Service social

\title{
Représentations sociales du mariage endogame et de ses conséquences biologiques sur la santé des descendants chez des fiancés apparentés
} Cas de deux villages chiites au Lilban

\section{Foumia Bou-Assy, Serge Dumont et Francine Saillant}

Volume 50, numéro 1, 2003

URI : https://id.erudit.org/iderudit/006994ar

DOI : https://doi.org/10.7202/006994ar

Aller au sommaire du numéro

Éditeur(s)

École de service social de l'Université Laval

ISSN

1708-1734 (numérique)

Découvrir la revue

Citer cet article

Bou-Assy, F., Dumont, S. \& Saillant, F. (2003). Représentations sociales du mariage endogame et de ses conséquences biologiques sur la santé des descendants chez des fiancés apparentés : cas de deux villages chiites au Liban. Service social, 50(1), 174-198. https://doi.org/10.7202/006994ar
Résumé de l'article

Cet article présente les résultats d'une étude portant sur les représentations sociales de l'endogamie et de ses conséquences biologiques sur la santé des descendants chez des fiancés apparentés musulmans chiites. La population à l'étude est issue de deux villages libanais. Des entrevues en profondeur ont été réalisées auprès de fiancés, hommes et femmes, d'informateurs clés et d'un groupe de mères. L'analyse qualitative de leur discours nous a permis de définir deux modèles de représentations sociales de l'endogamie : le mariage sécuritaire et le mariage risqué. Quant aux représentations sociales des conséquences biologiques de l'endogamie, nous avons identifié des causes d'ordre surnaturel, biologique, sociocomportemental et environnemental. Les résultats ont mis en évidence que l'endogamie, qui est une pratique sociale privilégiée dans les deux villages étudiés, ne peut être comprise qu'à la lumière de la culture et du contexte socio-économico-politique de ces deux collectivités. 


\section{Représentations sociales du mariage endogame et de ses conséquences biologiques sur la santé des descendants chez des fiancés apparentés}

\section{Cas de deux villages chiites au Liban}

Foumia Bou-Assy

Chargée de cours

École de service social de l'Université de Montréal

Superviseure mobile École de service social de l'Université Laval Organisatrice communautaire Centre de santé de la Haute-Saint-Charles Courriel : foumia@hotmail.com

Serge Dumont

Professeur agrégé École de service social de l'Université Laval Courriel : serge.dumont@svs.ulaval.ca

Francine SAILLANT

Professeure titulaire Département d'anthropologie de I'Université Laval Courriel : francine.saillant@ant.ulaval.ca

Cet article présente les résultats d'une étude portant sur les représentations sociales de l'endogamie et de ses conséquences biologiques sur la santé des descendants chez des fiancés apparentés musulmans chiites. La population à l'étude est issue de deux villages libanais. Des entrevues en profondeur ont été réalisées auprès de fiancés, hommes et femmes, d'informateurs clés et d'un groupe de mères. L'analyse qualitative de leur discours nous a permis de définir deux modèles de représentations 
sociales de l'endogamie : le mariage sécuritaire et le mariage risqué. Quant aux représentations sociales des conséquences biologiques de l'endogamie, nous avons identifié des causes d'ordre surnaturel, biologique, sociocomportemental et environnemental. Les résultats ont mis en évidence que l'endogamie, qui est une pratique sociale privilégiée dans les deux villages étudiés, ne peut être comprise qu'à la lumière de la culture et du contexte socioéconomico-politique de ces deux collectivités.

Mots clés : mariage, consanguinité, représentation sociale, ruralité

This article gives the results of a study held on the social representation of endogamy and of its biological consequences on the health of the descendants of Shiite Muslims engaged in endogamous couples. The people studied come from two Lebanese villages. Interviews were held with engaged couples, key informers and a group of mothers. Qualitative analysis of what they said let us identify two models of social representation of endogamous marriages: the safe one and the risky one. As to social representations associated with the origins of the biological consequences of endogamous marriage, the causes could be defined precisely as supernatural, biological, sociobehavioral and environmental. The results of this research show that the endogamous marriage, which is the most frequently practiced in the two villages mentioned above, can only be conceived by means of cultural and socio-economico-political understanding of these two communities.

Key words: marriage, consanguinity, social representation, rural sociology 


\section{L'ENDOGAMIE DANS LE MONDE ARABE ET ISLAMIQUE}

II est utile, tout d'abord, de préciser qu'il existe différents types d'endogamie : l'endogamie familiale, l'endogamie interreligieuse, l'endogamie intercommunautaire et l'endogamie spatiale (LéviStrauss, 1977). Dans la présente recherche, le terme « endogamie » désigne l'endogamie familiale. Le mariage est dit endogame lorsque les conjoints ont un ou des ancêtres communs. Le mariage avec la cousine parallèle patrilinéaire constitue la première forme d'endogamie possible. Des écarts s'affirment entre les cousins, les patrilatéraux sont valorisés en raison de la hiérarchie sexuelle. Ces écarts varient en fonction du type de mariage réalisé par les parents : si la sœur du père a épousé un agnat, il est certain que ses enfants entretiendront avec les membres de la fratrie des liens fondés sur une identité agnatique renforcée, ce qui ne sera pas le cas si elle se marie en dehors du groupe agnatique. II est remarquable que le statut des cousins croisés dépende entièrement des mariages de la génération précédente (Lévi-Strauss, 1977). En réalité, dans les sociétés arabes, toutes les catégories de cousins s'épousent entre elles (Conte, 1987), bien que le mariage entre les cousins parallèles patrilinéaires soit reconnu par le « mariage arabe ». Selon Breteau et ses collaborateurs (1981), on peut épouser toute cousine sans cesser d'être endogamique. Dans cette recherche, le mariage que nous qualifions d'endogamique est de type patrilinéaire ainsi que matrilinéaire.

L'endogamie, qui est une particularité du système des alliances dans le monde arabe et islamique, est encore contractée en Jordanie, en Palestine, en Syrie, en Iraq, au Koweït, en Arabie saoudite, au Kurdistan, en Iran, en Égypte, au Soudan, en Afrique du Nord et au Liban (Chelhod, 1965; Khlat, 1989; Lamdouar Bouazzaoui, 1994). L'endogamie est d'origine préislamique puisque les sociétés arabes l'ont constamment privilégiée, avant comme après la révélation coranique. Les études effectuées sur cette pratique ont révélé qu'elle est toujours en vigueur dans les milieux ruraux.

L'endogamie peut entraîner des conséquences sur la santé des descendants: elle augmente, entre autres, l'incidence des 
maladies génétiques (Der Kaloustian, dans Mustapha, 1997). En effet, le développement des connaissances en génétique humaine et en épidémiologie a permis l'identification de maladies dites génétiques. De ces mêmes connaissances découle la notion de risque. Selon Briard et ses collaborateurs (dans Mustapha, 1997), le risque dépend de deux catégories de facteurs: le lien de parenté entre les conjoints et l'existence dans la famille d'affections héréditaires récessives autosomiques ou multifactorielles. Les auteurs avancent également que la consanguinité ne crée pas de gènes pathogènes, mais qu'elle les associe plus fréquemment. Au Liban, les travaux réalisés par Der Kaloustian, Naffah et Loiselet (1980) ont ouvert d'importantes voies de recherche sur les désordres génétiques récessifs autosomiques. Ces chercheurs ont en effet publié une liste de trente-six maladies autosomiques récessives rares diagnostiquées et reconnues pour la première fois dans le pays. Les taux élevés de consanguinité dans les différentes confessions religieuses libanaises seraient à l'origine de la propagation des maladies autosomiques récessives observées.

La consanguinité semble accroître le taux de malformations congénitales telles que les cardiopathies et les néphropathies (Mustapha, 1997; Aoun, 1995; El-Kazen, 1993), l'incidence de la surdi-mutité (Akl, 1994), de la cécité (OMS, 1993) ainsi que des maladies génétiques comme l'encéphalopathie et certaines affections hématologiques (Mustapha, 1997) ou maladies rares (Der Kaloustian, dans Mustapha). Sur le plan humain, ces maladies constituent un sérieux problème médical et social du monde arabe, en particulier lorsqu'elles se traduisent par des déficiences et des incapacités évolutives. Le tableau 1, réalisé à partir d'une recension d'écrits sur le phénomène, donne un bref aperçu de la situation dans les pays méditerranéens et arabes. 
Tableau 1

Quelques conséquences biologiques de la consanguinité dans des pays méditerranéens et arabes

\begin{tabular}{|c|c|c|c|c|}
\hline Pays & Maladie & $\begin{array}{l}\text { Taux de } \\
\text { consan- } \\
\text { guinité } \\
(\%)\end{array}$ & Étude & Année \\
\hline Algérie & $\begin{array}{l}\text { Diabète juvénile } \\
\text { Polymalformation } \\
\text { Affections hémato- } \\
\text { logiques }\end{array}$ & $\begin{array}{l}27,77 \\
50,00 \\
39,32\end{array}$ & $\begin{array}{l}\text { Bénallègue } \\
\text { et Kedgi }\end{array}$ & 1984 \\
\hline Tunisie & Diabète juvénile & 48,00 & $\begin{array}{l}\text { Mongalgie } \\
\text { et al. }\end{array}$ & 1991 \\
\hline Tunisie & $\begin{array}{l}\text { Glaucome } \\
\text { Cataracte } \\
\text { Dégénérescence } \\
\text { Rétino-vitréenne }\end{array}$ & $\begin{array}{l}64,60 \\
65,50 \\
50,50\end{array}$ & Ayed et al. & 1991 \\
\hline Chypre & Cécité & 79,00 & OMS & 1993 \\
\hline Liban & Cécité & 68,00 & OMS & 1993 \\
\hline Liban & $\begin{array}{l}\text { Cardiopathie congé- } \\
\text { nitale }\end{array}$ & 17,50 & $\begin{array}{l}\text { El-Kazen } \\
\text { et al. }\end{array}$ & 1993 \\
\hline Liban & Surdi-mutité & 29,20 & Akl & 1994 \\
\hline Liban & $\begin{array}{l}\text { Insuffisance rénale } \\
\text { chronique }\end{array}$ & 26,00 & Aoun et al. & 1995 \\
\hline
\end{tabular}

Étant donné l'ampleur du phénomène du mariage endogame dans le milieu rural arabe et la gravité de ses conséquences sur les descendants, un comité de la Ligue arabe sur la médecine génétique s'est réuni en octobre 1995 au Caire avec les représentants des ministres de la santé d'Égypte, d'Arabie saoudite, de la Syrie, de la Tunisie, du Koweït, de la Jordanie, de Bahreïn et des Émirats arabes unis pour étudier les possibilités de la création d'une carte régionale génétique en vue de lutter contre les maladies héréditaires liées au mariage endogame (Journal de Québec, 16 octobre 1995, p. 13). 
Au Liban, la loi 334 promulguée en 1994 oblige les fiancés à se procurer un certificat médical prénuptial (EMP) afin de diminuer la fréquence des maladies génétiques et héréditaires. Dans ce certificat, les résultats des examens médicaux cliniques et des prélèvements de sang où l'hémoglobine, l'hématocrite, le MCV, le groupe rhésus, le HIV, et l'hémoglobine électrophorèse si le taux d'hémoglobine est inférieur à $70 \%$, sont consignés. D'autres examens sont exigés au besoin, par exemple pour la rubéole, le VDRL, le PPD, la toxoplasmose et l'hépatite B. II est également demandé au médecin d'expliquer aux fiancés apparentés l'importance de consulter un généticien et d'avoir un conseil génétique. Bien qu'une loi similaire ait été adoptée en 1956 en Tunisie, le taux de maladies génétiques autosomiques récessives y est demeuré élevé, surtout en milieu rural.

\section{UN BREF APERÇU DES DEUX VILLAGES : CHMISTAR ET TARAYYA}

Afin de mieux mettre en contexte la problématique de l'endogamie et de ses conséquences biologiques sur la santé des descendants en milieu rural libanais, nous présentons très brièvement les deux villages, Chmistar et Tarayya, où l'étude a été effectuée. II s'agit de villages musulmans chiites situés à l'ouest de la région de Baalbeck. À l'intérieur de chacun de ces villages, toute personne a son lignage comme cadre de référence et, en dehors de celui-ci, son appartenance revient au village. L'esprit de solidarité et d'entraide caractérise la réalité villageoise. Ainsi, tous les habitants se connaissent et les rapports sociaux sont d'une grande proximité. Les responsabilités de la femme tournent autour de son foyer conjugal et de sa famille. II n'est pas facile pour elle d'accéder au monde politique et les possibilités d'études avancées ou de travail rémunéré sont rares. Le mariage est fortement recommandé. D'ailleurs, l'islam, la religion d'appartenance des habitants de Chmistar et de Tarayya, propose à la femme et à l'homme un modèle à suivre et leur suggère des attitudes sociocomportementales. Les deux villages souffrent d'un manque de services spécialisés et d'un déclin attribuable à l'exode des jeunes travailleurs et diplômés, à la déstructuration des économies traditionnelles fondées principalement sur l'agriculture et à la perte de confiance en l'avenir de la région. 
La présente étude a pour but d'explorer le savoir des fiancés apparentés musulmans chiites sur le mariage endogame et ses conséquences biologiques sur la santé des descendants. De façon plus précise, l'étude se propose, à partir des discours de ces fiancés, de comprendre ce que ces derniers savent du mariage endogame, de dépeindre les images qu'ils ont de la parenté et du cousin et de découvrir leurs attitudes à l'égard du mariage endogame. L'étude tente également de comprendre à quoi ces fiancés attribuent l'atteinte, par une maladie génétique, d'un enfant dont les parents sont apparentés, ce qu'ils savent des systèmes thérapeutiques et préventifs et de découvrir leurs attitudes à l'égard de l'examen médical prénuptial.

\section{LE CADRE THÉORIQUE}

La théorie des représentations sociales a été retenue à titre de cadre théorique et conceptuel aux fins de la réalisation de la présente recherche qui repose essentiellement sur un devis qualitatif. L'étude des représentations sociales qui se rapportent à l'endogamie et à ses conséquences biologiques sur la santé des descendants sera au centre de la démarche scientifique dans la mesure où elle apportera un éclairage susceptible de favoriser la compréhension de l'endogamie en tant que pratique sociale ainsi que les systèmes étiologiques, thérapeutiques et préventifs qui lui sont associés. L'analyse s'attachera spécifiquement à l'examen d'une théorie populaire de ce que sont l'endogamie et ses conséquences biologiques sur la santé des descendants, comme à l'analyse de leur formation dans un contexte social donné. La démarche suivie se situe dans la ligne tracée par Moscovici (1961) dans son étude sur la psychanalyse, son image et son public, ainsi que par Jodelet (1989) sur les folies et les représentations sociales. Ces études sont centrées sur la façon dont les représentations sociales, en tant que "théories " socialement créées et opérantes, sont liées avec la construction de la réalité quotidienne, les conduites et les communications qui s'y développent. La théorie des représentations sociales suppose qu'il n'y a pas de coupure entre l'univers extérieur et l'univers intérieur de l'individu ou du groupe (Moscovici, dans Abric, 1994). Ainsi, selon Mannoni (1998), les représentations sociales, situées à la jonction du psychologique et 
du sociologique, s'enracinent au cœur du dispositif social et sont l'élément saillant et signifiant du discours.

\section{LE CADRE MÉTHODOLOGIQUE}

Nous avons choisi les deux villages musulmans chiites Chmistar et Tarayya, situés dans la région de Baalbeck, car on y trouve l'un des taux les plus élevés de mariages endogames au Liban.

\section{Tableau 2}

La distribution de tous les fiancés à Chmistar et à Tarayya en janvier 1998 selon le type de fiançailles

\begin{tabular}{|l|c|c|l|c|c|}
\hline $\begin{array}{l}\text { Types de } \\
\text { fiançailles } \\
\text { à Chmistar }\end{array}$ & $\mathbf{N}$ & $\%$ & $\begin{array}{l}\text { Types de } \\
\text { fiançailles } \\
\text { à Tarayya }\end{array}$ & $\mathbf{N}$ & $\%$ \\
\hline Cousins germains & 14 & 16,47 & Cousins germains & 8 & 18,60 \\
\hline Parenté éloignée & 22 & 25,88 & Même lignage & 5 & 11,62 \\
\hline Pas de parenté & 27 & 31,76 & Même patronyme & 17 & 39,53 \\
\hline $\begin{array}{l}\text { À l'extérieur } \\
\text { du village }\end{array}$ & 22 & 25,88 & $\begin{array}{l}\text { À l'extérieur } \\
\text { du village }\end{array}$ & 13 & 30,23 \\
\hline Total & 85 & 99,99 & Total & 43 & 99,98 \\
\hline
\end{tabular}

Le tableau ci-dessus montre qu'à Chmistar le taux de fiançailles avec les cousins germains et la parenté éloignée correspond approximativement aux deux cinquièmes $(42,35 \%)$ du nombre total des fiançailles. À Tarayya, l'autre village, qui comprend treize lignages issus d'un seul patronyme, les deux tiers des fiancés ont choisi un partenaire du même patronyme. La stratégie de recherche utilisée est de type qualitatif. Les données proviennent principalement d'entrevues semi-structurées réalisées auprès de 30 fiancés, d'un groupe de mères et de neuf informateurs clés (deux chefs religieux, deux médecins généralistes, un médecin généticien, deux biologistes, deux travailleuses sociales). II s'agit de 30 fiancés cousins germains (issus de deux germains de même sexe, deux frères ou deux sœurs, ou issus de germains de sexe différent, un frère et sa sœur), patrilinéaires (du côté du père) ou matrilinéaires (du côté de la mère) bilatéraux (du côté du père et 
de la mère). Ces fiancés viennent tous de la communauté musulmane chiite, puisque tous les habitants de Chmistar et de Tarayya sont des Chiites, qui forment donc une population relativement homogène, ayant à partager un même dogme islamique. Un guide d'entrevue a été élaboré pour chacune des catégories d'informateurs et pour le groupe témoin de mères. Ces guides comprenaient des questions ouvertes visant, entre autres, à faire parler les fiancés et les informateurs clés sur ce qu'ils savent du mariage endogame et de ses conséquences biologiques sur la santé des descendants.

Les 40 entrevues, menées en profondeur, ont été effectuées sur une période de trois mois, entre janvier et mars 1998. Ces entrevues ont été enregistrées à l'aide d'un magnétophone, sauf celles de deux fiancés qui ont refusé l'enregistrement audio. Le matériel a été transcrit intégralement en arabe et ensuite traduit en français. Seulement deux entrevues se sont faites en français. Nous avons analysé ce matériel selon la théorisation ancrée élaborée en 1967 par Glaser et Strauss. II s'agit d'une analyse de type représentationnel, axée sur la présence des idées et non pas sur la fréquence. Trois grandes étapes marquent l'analyse par théorisation ancrée: la codification, la catégorisation et la mise en lien. Nous avons recouru à deux personnes originaires du village de Chmistar et qui demeurent à Québec pour la transcription textuelle, de même qu'à une linguiste d'origine libanaise qui a vérifié la traduction de deux entrevues choisies au hasard pour valider la traduction de l'arabe au français. Quant au processus de codage et de catégorisation, tout le travail de catégorisation a été fait de telle sorte que le jugement à l'égard des données à catégoriser soit uniforme.

\section{LES RÉSULTATS}

Dans cette partie, nous tenterons d'abord de cerner le mariage endogame et ses conséquences sur la santé des descendants, et ce, selon les trois dimensions constitutives de la représentation sociale, soit l'information, l'image et l'attitude, qui sont imbriquées les unes dans les autres. Ensuite, la construction socioculturelle de ce mariage et de ses conséquences sera analysée. 


\section{Les représentations sociales du mariage endogame}

Pour plusieurs des fiancés rencontrés dans le cadre de la présente recherche, l'endogamie est une pratique sociale préférentielle et normative. Elle est justifiée par eux du fait que le Prophète lui-même l'a pratiquée en vue d'accroître sa lignée, une préoccupation importante dans le monde islamique. Pour ces fiancés, le Prophète est l'exemple à suivre. L'endogamie garantit une continuité de la manière d'être et de la façon de faire, une protection de l'honneur de la femme, une stabilité de vie conjugale et une meilleure dynamique familiale, de même qu'une sécurité affective, psychologique et financière. Le fiancé n'a pas à s'intégrer dans un nouveau milieu ni à faire des efforts pour répondre aux exigences de sa fiancée: "Je connais exactement cette maison [famille]. Précisément, je connais cette jeune fille et ses comportements. Nous ne pouvons frapper la porte que de celui que nous connaissons, c'est rare... » $(3, \mathrm{H})^{1}$. Étant du milieu, la cousine est habituée aux travaux et au mode de vie de ses beaux-parents. Ensemble, les fiancés partagent le même désir de posséder une maison, car être propriétaire de biens en général, et d'une maison en particulier, est dans la mentalité rurale une nécessité et un moyen de garantir la sécurité matérielle. À l'opposé, l'incertitude existe de part et d'autre avec la femme étrangère, qui est perçue comme plus exigeante et indifférente aux conditions matérielles dans lesquelles vit son fiancé.

L'endogamie constitue une pratique également bénéfique pour les parents et la collectivité et une forme d'entraide entre les familles. Elle renforce les liens interfamiliaux et intrafamiliaux d'un même groupe. Selon Bourdieu (1980), l'endogamie a pour effet de contribuer de façon déterminante à créer un groupe intégré et de limiter sa tendance au fractionnement. La façon dont les comportements de la personne apparentée et des beaux-parents sont décrits et justifiés laisse émerger des sentiments de joie et de confiance.

Par ailleurs, l'exogamie semble affaiblir l'appartenance au groupe : «Si quelqu'un se marie en dehors du village, on dit qu'il

1. Nous identifions les fiancés comme étant des répondants ou des répondantes et inscrivons à la suite leur genre et leur code. 
est sorti de sa famille. Par principe, il ne doit pas se marier avec une fille qui n'est pas de son village. La mentalité des gens est toujours la même à l'égard du mariage endogame » $(16, \mathrm{H})$. La règle d'exogamie distingue nettement des groupes d'alliance et des groupes de filiation qui, par définition, ne peuvent coïncider.

Un médecin $(34)^{2}$ évoque les conséquences du mariage endogame sur le couple, qui seraient d'ordre psychologique, relationnel et sexuel, et les conséquences sur leur famille. Ces dernières sont surtout d'ordre relationnel :

L'endogamie est un repli sur soi [...]. Quelquefois, la nuit de noces, le nouveau marié ne joue pas son rôle puisqu'il s'est habitué à une image autre que celle de la mariée [...]. C'est une question plutôt psychologique qu'organique. Cette femme, future partenaire, dans son inconscient était celle avec qui il jouait tout au long de son enfance. Ceci est un facteur d'échec du mariage et non pas de réussite comme les parents imaginent [...]. Même, les problèmes sociaux sont accentués, tels que des conflits déjà existants entre les parents. L'endogamie est un milieu propice pour l'intervention des parents et l'intensification de leur querelle (34).

Certaines répondantes confirment en quelque sorte le témoignage du médecin. Elles soulèvent les conséquences psychologiques du mariage endogame sur le couple, telles que l'absence de nouveautés et de curiosités qui entravent le plaisir de connaître et de découvrir l'autre, l'amour du partenaire qui est un amour fraternel : " Au fond, je n'aime pas me marier avec un cousin maternel ou paternel. J'aime plus l'homme étranger... » $(28, \mathrm{~F})$. Ces fiancés expriment d'une manière subjective le privilège du mariage exogamique et le désir profond de se marier avec un étranger. Au repliement du groupe sur soi-même, et aussi sur son propre passé, sur ses traditions s'oppose l'ouverture vers l'extérieur et vers le nouveau.

Les fiancés considèrent que les cousins et les cousines sont des frères et sœurs. Ils désignent distinctement les frères et sœurs de leur père et de leur mère sous les noms de " oncle paternel ",

2. Un chef religieux, un médecin, un biologiste, une travailleuse sociale sont vus comme étant des informateurs clés. Nous inscrivons leur code entre parenthèses à la suite de leur dénomination. 
" oncle maternel » et "tante paternelle », "tante maternelle ». L'oncle paternel est assimilé au père par le sang et la tante maternelle est assimilée à la mère par l'affection. Du côté paternel, il s'agit d'une parenté légitime qui est basée sur le lien de sang. Du côté maternel, la tendresse, la familiarité, la simplicité et le sentiment d'être à l'aise sont prépondérants. La tante maternelle est cependant une parente plus éloignée que l'oncle paternel, puisque son mari est un étranger. La femme et l'homme jouent donc différents rôles dans la transmission à leur descendant : le rôle de la femme est de transmettre l'affection, celui de l'homme, le sang. Ainsi, selon le groupe des mères, les interactions et les liens sont distincts entre la parenté patrilinéaire et la parenté matrilinéaire et, par conséquent, entre les cousins patrilinéaires et matrilinéaires: "Le cousin parallèle patrilinéaire, c'est la lignée, la parenté de sang, c'est le même sang. Le cousin maternel, c'est l'affection » (38).

Le cousin serait compréhensif, plus attentionné et indulgent à l'égard de sa cousine. II reste le même pour le meilleur et le pire, et fournit la stabilité matérielle et affective. Quant à la cousine, elle est également plus attentionnée et permissive avec son fiancé et ses beaux-parents, et se caractérise par sa tolérance et sa flexibilité. Elle inspire confiance, admet le doux et l'amer et comprend mieux, selon les répondants, leur situation personnelle et familiale. Dans les pires moments, elle sait s'adapter et reste à leurs côtés. Pour certains fiancés, c'est le lien fraternel qui se transforme en un lien conjugal; par contre, pour d'autres, c'est plutôt le lien amical qui se transforme en un lien conjugal. Les discours des fiancés sur leurs fiançailles ont permis de comprendre que les parents jouent un rôle décisif dans le mariage de leur fils ou de leur fille. Quand il s'agit du mariage précoce de la fille, leur rôle s'accentue. En général, les parents contrôlent le choix de l'éventuel partenaire de leur enfant. La mère, en particulier, exerce une grande influence dans le choix de sa future bru. Lorsque le père accorde la main de sa fille à un jeune homme, celle-ci peut difficilement refuser; elle doit accepter son sort, sa marge de manœuvre étant assez limitée. Parfois, la vie de la fille peut être menacée, si elle se marie sans l'aval de ses parents. 
Les deux chefs religieux indiquent qu'ils n'interdisent pas le mariage endogame; ils conseillent cependant de l'éviter. Les directives des chefs religieux sont appuyées par le point de vue des autorités sanitaires. Un biologiste affirme catégoriquement qu'aucune autorité n'a le droit d'interdire à qui que ce soit le mariage avec la personne désirée. Quant à son confrère (34), qui est né dans l'un des villages à l'étude et y pratique la médecine, il exprime sa sympathie pour le mariage endogame, bien qu'il affirme être conscient de ses conséquences. II révèle que cette forme de mariage est ancrée dans le cœur et l'esprit.

La référence au champ normatif est très présente dans le discours des fiancés: "C'est à la mode de se marier entre les cousins parallèles patrilinéaires » $(30, F)$ et "C'est normal de se marier avec quelqu'un de la parenté » $(27, \mathrm{~F})$. «C'est normal » va dans le sens de l'ancrage chez les fiancés: «Dès que j'ai eu l'idée du mariage, je n'ai pensé qu'à me marier avec une fille de la parenté. II n'y a rien à comparer entre la fille parente et la fille étrangère... » $(11, H)$. La majorité des fiancés rencontrés ont exprimé une attitude très favorable à l'égard du mariage endogame. Par contre, ils expriment être défavorables au mariage avec un cousin bilatéral et au choix matrimonial qui serait imposé par leurs parents. Ces derniers encouragent leur mariage avec le cousin ou la cousine et espèrent que leurs autres enfants pratiquent cette forme de mariage.

Le discours des fiancés nous a permis de concevoir deux modèles de représentations sociales du mariage endogame, soit: le mariage "sécuritaire » et le mariage "risqué ". Le mariage sécuritaire qui fait référence au mariage entre cousins patrilinéaires ou matrilinéaires serait le mariage idéal lorsque le partenaire est convenable. Des dictons populaires illustrent bien cette préférence : "Celui qui choisit en dehors de son clan rencontrera des malheurs autres que les siens » $(16, \mathrm{H})$. L'attitude des fiancés et de leurs parents est très favorable à ce type de mariage. Quant au mariage risqué, il fait référence au mariage entre cousins bilatéraux. Ce mariage suscite des peurs, puisqu'il est à haut risque des conséquences biologiques sur la santé des descendants. L'attitude des fiancés interviewés sur cette forme de mariage est foncièrement défavorable. 


\section{Les représentations sociales des conséquences biologiques du mariage endogame sur la santé des descendants}

Les conséquences biologiques de l'endogamie sur la santé des descendants sont un sujet délicat de la présente recherche. Plusieurs fiancés rencontrés et quelques représentantes du groupe de mères ont éprouvé de la difficulté à répondre à la question suivante: "Pouvez-vous me mentionner des cas d'enfants atteints par une maladie congénitale et incurable ou d'un handicap fonctionnel que vous connaissez ou dont vous avez entendu parler et dont les parents sont des cousins germains? "Cette question a plusieurs fois suscité un inconfort, de la gêne, de la réticence, en plus de provoquer de longs silences. Elle a éveillé des peurs refoulées, rappelé les expériences familiales et les histoires tristes transmises par l'entourage. Plus d'un tiers des fiancés ont témoigné de l'existence d'enfants atteints d'une maladie congénitale ou d'une déficience dans leur fratrie ou leur parenté immédiate et presque un tiers ont révélé des cas dans leur voisinage. Également, deux femmes du groupe des mères qui sont mariées à leur cousin patrilinéaire ont des enfants souffrant de maladies congénitales. Pour les médecins et les biologistes, l'endogamie contribue à l'augmentation du nombre de personnes affectées par les maladies récessives. Cependant, l'un de ces médecins précise qu'il est difficile de démontrer aux gens qu'il existe des conséquences biologiques liées au mariage endogame et, par conséquent, de les empêcher de contracter cette forme de mariage.

Les discours des fiancés et du groupe des mères ont permis de regrouper six catégories de causes des maladies ou malformations chez les enfants. Ces causes présentées ici par ordre d'importance, selon ce qui a été observé auprès des sujets, sont les suivantes: les causes surnaturelles, biologiques, sociocomportementales, mixtes, environnementales et le non-savoir. Ce regroupement en catégories de causes permet de clarifier certaines tendances qui se sont dégagées de cette analyse.

La catégorie de causes d'ordre surnaturel, telles que la volonté de Dieu $(n=23)$ et les maléfices $(n=16)$, est la plus souvent évoquée. Dans le premier regroupement de causes, les notions 
comme " la puissance de Dieu », " tout est de Dieu », "le don de Dieu " sont des notions imprégnées dans l'imaginaire collectif des gens : "Dans cette société, c'est le même discours : la volonté de Dieu est avant tout » $(28, F)$. La responsabilité des parents apparentés, en particulier, et celle de l'être humain et de l'environnement, en général, sont ainsi écartées. En référence au Coran, l'idée du destin, qui a été exprimée fréquemment par les fiancés pour expliquer les conséquences biologiques, présume que la maladie est un don de Dieu. Les gens associent à des forces surnaturelles tout ce qui est inexpliqué, mystérieux: "Lorsque la personne ne réussit pas à comprendre les causes et les résultats d'un événement, elle a recours à ces forces pour s'apaiser et avoir l'âme tranquille » $(27, \mathrm{~F})$. Si une personne se moque d'un enfant atteint par un handicap, son enfant l'aura aussi : « Dieu l'a punie » $(30, \mathrm{~F})$. Même les personnes instruites peuvent croire que la volonté divine provoque la maladie et accepter l'existence des maléfices.

Dans le deuxième regroupement de causes d'ordre surnaturel, les maléfices tels que le mauvais œil, l'oppression, la sorcellerie et la possession provoquent des changements néfastes dans le bienêtre, les sentiments, les comportements ou la santé d'un nouveau-né. Ils causent même la mort de ce dernier. Les maléfices provoquent également l'avortement chez une femme enceinte et ont une action sur l'allaitement des mères. Le mauvais œil est mentionné dans le Coran ainsi que la méchanceté du jaloux. Un chef religieux confirme que «la religion croit en son existence, mais nous ne connaissons pas ses causes » (31).

En ce qui a trait à la catégorie de causes d'ordre biologique, elle occupe la deuxième position en importance. II s'agit de la consanguinité $(n=15)$ et de l'hérédité $(n=13)$. La référence à une étiologie caractérisée par la transmission de la maladie (hérédité, consanguinité) nourrit directement ou indirectement la culpabilité chez les parents : "Si la mère ou le père porte une maladie, leurs enfants ont une prédisposition à la porter » $(2, \mathrm{~F})$, "Quand on se marie trop dans la famille... » $(23, \mathrm{~F})$. II s'agit ici d'une association particulièrement directe entre la maladie génétique et l'endogamie. Nous sommes devant un discours qui s'approche du discours médical ou qui s'inscrit dans ce discours. II semble ici que le 
discours savant ait pénétré peu à peu le discours populaire. Le groupe des mères souligne l'influence du savoir savant dans la modification de leur savoir, mais celle-ci est davantage perceptible lorsque les informations médicales ne sont pas en contradiction avec ce qui est écrit dans le Coran : "Ce qui est dans le Coran est absolu. ॥

La catégorie de causes d'ordre sociocomportemental fait référence au comportement sexuel du père ou de la mère $(n=9)$. Sur ce point, les discours des fiancés et du groupe des mères renvoient aux enseignements religieux qui prescrivent les normes de bonne conduite en matière de sexualité. Ainsi sont proscrites les relations sexuelles prénuptiales et les relations sexuelles durant la période des règles alors que la femme est impure et que son sang peut être en contact avec la semence du mari. Enfin, l'omission par le mari de réciter un verset coranique avant une relation sexuelle constitue une transgression aux règles. L'individu est alors pénalisé dans sa progéniture lorsqu'il ne respecte pas les règles édictées par la doctrine religieuse. Au chapitre des causes de nature sociocomportementale, le discours des sujets met en cause l'utilisation des médicaments et plus particulièrement l'usage de contraceptifs.

La présence d'une étiologie mixte $(n=6)$, biologique et surnaturelle, est particulièrement intéressante. On y reconnaît certains déterminants biologiques associés à l'hérédité et à la consanguinité : "la science a une influence, mais Dieu a une influence décisive dans les malformations » $(8, H)$. Dans un milieu où la religion est prééminente, la coexistence de cette dernière avec la science biomédicale en développement met en relief la complexité des conséquences biologiques associées à la consanguinité pour les jeunes fiancés. Elle signale une ambivalence liée à l'existence d'une tension entre le monde religieux et le monde scientifique. II n'est pas facile pour le croyant musulman de ne recourir qu'à la science et d'oblitérer les enseignements religieux pour comprendre la cause de la maladie.

La catégorie de causes de nature environnementale $(n=4)$ relève de la modernité et concerne des phénomènes contemporains comme la pollution, la présence des produits chimiques, etc. On 
dira alors que la " nourriture est intoxiquée », " tout est pollué ». Enfin, quelques sujets $(n=4)$ indiquent leur difficulté à se faire une opinion sur l'étiologie des maladies congénitales observées chez les enfants dont les parents sont apparentés. On perçoit chez eux de l'ambivalence et de l'incertitude, voire un inconfort : "On ne sait pas si c'est de Dieu ou si ce n'est pas de Dieu ».

Les systèmes étiologiques dégagent des représentations divines, maléfiques, biologiques, génétiques, sociales, environnementales des conséquences biologiques de la consanguinité chez les descendants et placent les différents types d'atteinte sur un continuum dont les pôles extrêmes sont le surnaturel et l'humain. Cependant, de ces systèmes étiologiques découle un recours à des thérapies reconnues médicalement ou associées au discours savant et à des thérapies traditionnelles issues du savoir populaire. Dans un même ordre d'idées, des rituels religieux et maléfiques se combineront avec les enseignements des médecins pour traiter la maladie génétique ou la prévenir.

Quant aux systèmes préventifs, il est utile de rappeler que l'examen médical prénuptial (EMP) et le conseil génétique sont des mesures mises en place dans le but de réduire le nombre de maladies génétiques. Pour plusieurs fiancés, l'examen médical prénuptial n'est qu'un prélèvement de spécimens de sang de la jeune fille et du jeune homme. En effet, le couple se fait ausculter par le médecin et il se fait faire un examen de sang afin d'obtenir un certificat médical qu'il présentera au tribunal religieux. Presque le quart des fiancés n'ont aucune idée de ce qu'est l'EMP; ils ne se sont pas renseignés par manque de curiosité ou parce que, selon eux, il est encore tôt pour passer l'examen. Un répondant ne sait pas quoi en dire. Certains n'ont pas encore soulevé cette question avec leur partenaire. Une répondante indique que c'est la première fois qu'elle entend parler de cet examen. En effet, ses trois sœurs déjà mariées ne l'ont pas subi. Une autre répondante, enfin, ne sait même pas comment cet examen est "nommé »; c'est quelque chose de nouveau pour elle.

L'examen médical prénuptial apparaît comme une tâche lourde, mais il empêche rarement la réalisation du projet de mariage et reste une simple formalité administrative. C'est l'homme qui va 
surtout s'informer du " comment le subir ". Si certains fiancés ne s'y intéressent pas, d'autres le trouvent important, tandis que d'autres remettent son utilité en question. Cet extrait est très significatif à cet égard: " malgré l'obligation de cet examen, des enfants naissent handicapés » $(19, F)$. Les fiancés se représentent l'EMP comme un examen susceptible de détecter tout risque de maladie ou de malformation chez la progéniture éventuelle, de vérifier la compatibilité sanguine et de prédire tous les problèmes susceptibles de survenir durant la grossesse et au moment de l'accouchement. L'approche de l'examen constitue une période anxiogène pour les fiancés, mais ses conclusions, généralement positives dans la grande majorité des cas, nourrissent un profond sentiment de sécurité au regard du futur.

L'explication de l'examen tourne autour du sang: propreté (absence de maladies) et compatibilité (groupe rhésus). En effet, du point de vue des fiancés, la maladie se transmet par le sang, elle est attribuable à sa mauvaise qualité et à son incompatibilité. Dans la population, on voit le sang et l'on en parle en termes de " même sang " et " sang différent ». Ainsi, la similitude et la différence constituent les deux pôles du sang. Pour les uns, la fraternité du sang garantit sa pureté et suffit à éliminer les menaces de la maladie. Similitude et identité autorisent l'union entre deux personnes apparentées. Même si le savoir médical sur le sang est limité, confus et parfois inexistant, autour du sang (similitude ou différence) s'organise tout un halo de significations qui porte un éclairage primordial sur la liaison entre le mariage et l'atteinte par une pathologie congénitale ou une malformation. Les fiancés croient que l'examen du sang est très significatif et révélateur.

Par ailleurs, les médecins expliquent qu'ils ont le sentiment d'être mis devant un fait accompli, car les fiancés expriment souvent une prise de position qui est de se marier peu importe les résultats de l'examen. Dans les faits, il semble bien que l'EMP empêche rarement la réalisation d'un projet de mariage et demeure une simple formalité administrative qui procure, par ailleurs, un grand sentiment de sécurité aux fiancés. Ceux-ci lui attribuent en effet une très grande capacité prédictive, et cela, malgré la portée tout à fait limitée d'un tel examen. Ainsi, une mesure scientifique et étatique 
peut être détournée de ses fins et servir à conforter une pratique proscrite.

L'étude révèle en outre que le conseil génétique est une mesure méconnue des fiancés et du groupe des mères. Même au sein du réseau de la santé, tous ne connaissent pas l'existence des deux laboratoires génétiques situés à Beyrouth. Les professionnels de ces laboratoires indiquent qu'il est souvent difficile de faire comprendre à des parents que la maladie de l'un de leurs enfants est d'origine génétique lorsque les autres enfants sont en bonne santé. Enfin, comme les frais associés à ces consultations ne sont pas payés par le ministère de la Santé, cela contribue à réduire l'accessibilité à ces dernières.

\section{La construction socioculturelle du mariage endogame et de ses conséquences sur la santé des descendants}

Le mariage endogame s'organise autour des distinctions exprimées entre la cousine et la femme étrangère. La réticence manifestée par la collectivité à l'égard de l'étrangère laisse à penser qu'il existe une différence fondamentale entre elle et la cousine. Par le biais des rapports intergénérationnels se diffusent et s'ancrent les modèles idéaux de la bru ou du beau-fils. Les processus par lesquels s'opèrent la transmission des règles et leur transformation en système normatif mettent en évidence la prévalence du contrôle social en matière d'adaptation à la vie quotidienne avec les beaux-parents. En même temps qu'elle donne corps par ses barrières concrètes ou symboliques à la différenciation de l'étiquette "étrangère ", la règle de l'écart pose les limites de l'intégration qu'autorise le bon accomplissement du rôle de " cousin ». Les modèles du mariage endogame que nous avons identifiés comme étant le mariage sécuritaire et le mariage risqué donnent une vue fidèle de la façon dont représentations et comportements sont liés, illustrant, par ses aspects structuraux et instrumentaux, les processus d'objectivation et d'ancrage dégagés dans la théorie des représentations sociales. Cela dit, les représentations sociales seraient en rapport avec les conduites et pourraient donc servir de guide d'action. La connaissance développée par la collectivité se fonde sur les expériences et sur les observations et s'oriente vers la pratique dont les règles se transmettent 
d'une génération à l'autre : par exemple, la relation de la bru parente avec ses beaux-parents, le traitement et la prévention des maladies chez l'enfant à naître. C'est aussi une connaissance construite à partir d'éléments divers et qui s'apparente au « bricolage » dont parle Lévi-Strauss à propos de la pensée sauvage (Jodelet, 1989).

Grâce aux connaissances transmises par les aïeux, les parents, le voisinage, les médecins, les enseignements religieux, les observations, les expériences et les informations médiatiques, les représentations sociales du mariage endogame et de ses conséquences biologiques se sont constituées et se sont maintenues. Les images se construisent et se codifient selon des conduites obligées, des normes sociales établies et exercent une pression à laquelle même les plus instruits de la collectivité finissent par se conformer. Ce processus de consolidation des manières de faire est accentué par la transmission de celles-ci d'une génération à l'autre. L'obéissance aux modèles imposés par les aînés, en vertu du principe d'autorité, est commandée et renforcée par la réussite d'expériences confirmées depuis longtemps. Les fiancés emploient un langage normatif : ils parlent de "règles ", de "principes ". C'est tout un protocole de savoir, de savoir-vivre, de savoir-être, de savoir-dire qui se transmet. En fait, partager une idée, un langage, c'est aussi affirmer un lien social et une identité collective.

II semble que cette recherche sur les représentations sociales ait permis de dégager des contenus susceptibles d'être mis en rapport, de démontrer que ces contenus sont cohérents, structurés et partagés. Autrement dit, leur construction ne se fait pas dans le désordre et l'adhésion collective à ces représentations est un moyen de s'affirmer dans son unité et son identité. Le savoir provenant des médias et de l'information médicale contribue peu à peu à élargir le champ explicatif de la maladie génétique au sein du savoir populaire. De plus, la modernité et ses impacts sur la qualité de l'environnement et son influence sur les modes de vie (surtout la liberté sexuelle) sont des facteurs de risque de plus en plus reconnus pour la santé des personnes. 
À l'instar de l'existence des causes d'ordre surnaturel et d'ordre biologique pour expliquer les conséquences biologiques de l'endogamie, il existe à Chmistar et à Tarayya un système thérapeutique savant et un système thérapeutique populaire pour traiter ces mêmes conséquences. Le premier système est accepté parce que le développement du savoir savant et la technologie sont parvenus à imposer leur crédibilité; le second l'est aussi, du fait que le mauvais œil, la sorcellerie, la possession et l'oppression font partie intégrante des systèmes étiologiques observés au sein de la culture de ces deux collectivités. C'est ainsi qu'à travers les représentations sociales des conséquences biologiques de la consanguinité, la présente recherche a pu montrer l'existence d'un système de pensée cohérent de la maladie, un savoir de sens commun qui est relativement indépendant du savoir savant. En effet, nous constatons l'existence même du discours populaire, concurrent du discours médical, et, surtout, son droit et sa pertinence pour parler des faits biologiques. Ces représentations, devenues en quelque sorte d'autant plus conscientes d'elles-mêmes, de leur spécificité et de leur valeur, veulent s'affirmer comme un discours collectif légitime.

En analysant comment cet ensemble de connaissances (bénéfices du mariage endogame), de valeurs (stabilité du mariage, cohésion et solidarité collective), d'images (cousin et cousine sont assimilés au frère et sœur), d'attitudes (positives à l'égard du mariage entre cousins paternels ou maternels) a été exprimé, vécu, pensé par les fiancés, nous constatons que les représentations sociales du mariage endogame et de ses conséquences biologiques s'élaborent, se structurent et se transforment sous l'influence de processus socioculturels et médicaux. Les possibilités de transformation de ces représentations apparaissent comme étroitement imbriquées dans des ensembles interdépendants d'attitudes, de croyances et de pratiques. À la lumière de cette analyse, le mariage endogame apparaît comme une pratique sociale difficile à changer et les conséquences biologiques de la consanguinité sur les descendants se révèlent être un phénomène médico-social très complexe. Ainsi, les intervenants sociaux sont appelés à porter une attention aux particularités socioculturelles des individus et des collectivités dans leur action et aux spécificités géographiques du milieu. 


\section{CONCLUSION}

Les bénéfices du mariage endogame sont appris et construits par l'intermédiaire de la socialisation et de la communication, à savoir la famille, la parenté et le voisinage. Cette forme de mariage offre aux couples et à leurs parents plus d'avantages que d'inconvénients sur les plans individuel, familial et collectif. Elle assure la sécurité affective et matérielle des conjoints, la stabilité du mariage, l'acceptation du partenaire et de ses parents dans leur être et leur avoir, le renforcement des liens interfamiliaux, la libération des parents du fardeau de leur enfant, la cohésion et la solidarité collective. Les fiancés partagent avec leur famille et leur entourage une représentation positive du mariage endogame lorsqu'il s'agit d'un cousin croisé patrilinéaire ou matrilinéaire, car les conséquences de la consanguinité seraient peu présentes dans ce type de mariage par comparaison avec le mariage entre cousins bilatéraux. De plus, l'examen médical prénuptial semble apaiser le doute quant aux conséquences biologiques possibles. En fait, l'expansion de ce mariage à travers l'histoire est attribuée aussi bien aux circonstances politiques qu'aux conditions socioculturelles du pays.

C'est ainsi que le mariage endogame, la conduite maritale privilégiée dans les deux villages, ne peut être compris qu'à la lumière de la culture et du contexte socio-économico-politique des deux collectivités. L'analyse des propos des fiancés nous a permis de constater que la question de la sécurité est très importante et que sa quête, qui constitue un élément explicatif du recours à l'endogamie - considérée socialement comme un objet sécuritaire -, s'accentue de plus en plus. La notion de sécurité renvoie en fait à l'unité microsociale du couple et du lignage et macrosociale de l'environnement et de la politique. II existe dans l'imaginaire des fiancés apparentés une relation entre leur santé et celle de leurs descendants. Cette relation est liée à la transmission du sang et à la croyance voulant que l'examen du sang permette de détecter tout risque de maladie. Par conséquent, les fiancés accordent beaucoup d'importance à cet examen et à ses conclusions.

L'examen médical prénuptial semble ainsi procurer une fausse sécurité aux personnes concernées. II contribue paradoxalement, 
malgré sa justification initiale, à assurer le maintien de la pratique du mariage endogame. Rappelons qu'une loi obligeant les fiancés à subir un examen médical prénuptial a été appliquée le 13 août 1956 en Tunisie et que le taux de maladies génétiques est demeuré élevé, surtout en milieu rural. L'obtention de «bons résultats » renforce l'idée du fatalisme et du déterminisme lorsque la maladie survient chez l'enfant. Les facteurs biologiques s'en trouvent ainsi minimisés.

Les représentations des conséquences biologiques de la consanguinité observées auprès des fiancés apparentés et du groupe des mères rencontrés dans le cadre de cette recherche permettent de comprendre le rôle des déterminants socioculturels dans l'analyse du mariage endogame, considéré comme un comportement à risque par la communauté scientifique. De plus, ces représentations permettent de mieux comprendre les réactions de ces personnes face aux programmes de prévention et de promotion mis en place par le gouvernement libanais il y a quelques années.

Le mariage endogame traduit fondamentalement une logique qui tire ses prémisses d'un savoir populaire permettant à l'individu de construire des systèmes explicatifs des causes, du développement et du traitement de la maladie et ne peut être compris que dans un système culturel, un contexte et une histoire déterminés. C'est pourquoi l'approche biomédicale traditionnelle s'ouvre progressivement aux déterminants psychosociaux et culturels de la santé. L'influence de la culture est à considérer dans la mise au point des mesures préventives, car elle peut constituer une barrière à l'actualisation de celles-ci. Des ponts sont nécessaires entre le savoir savant et le savoir populaire, tant pour ce qui est de la définition des besoins en services préventifs que pour le contenu des programmes de prévention et l'évaluation de leurs retombées. 


\section{RÉFÉRENCES BIBLIOGRAPHIQUES}

ABRIC, J.-C. (1994). Pratiques sociales et représentations, Paris, Presses universitaires de France.

AKL, E. (1994). Les étiologies de la surdité de l'enfant au Liban. Mémoire, Beyrouth, Faculté de médecine, Université Saint-Joseph.

AOUN, S., J. BOU-KHALIL et J. EL-NAHAS (1995). Effet de la consanguinité sur les maladies rénales au Liban : étude épidémiologique. Thèse, Beyrouth, Faculté des sciences médicales, Section II, Université libanaise.

Ayed, S., F. Daghfous, K. Guermazi et N. Ben Osman (1991). " Les causes de cécité de l'enfant tunisien », Trachoma pathologique oculaire tropicale - Subtrop - Santé publique, vol. 68, p. 123-128.

BÉnAlLĖGue, A. et F. KEDJI (1984). "Consanguinité et santé publique. Étude algérienne », Archives françaises de pédiatrie, vol. 41, p. $435-440$.

BONTE, P. (dir.) (1994). Épouser au plus proche: inceste, prohibitions et stratégies matrimoniales autour de la Méditerranée, Paris, EHSS.

Bourdieu, P. (1980). Le sens pratique, Paris, Éd. de Minuit.

Breteau, C-H., C. Lacoste-Dujardin, C. LefebVRe et N. Zagnoli (1981). Production-pouvoir et parenté dans le monde méditerranéen de Summer à nos jours, Paris, CNRS-EAESS.

CHELHOD, J. (1965). «Le mariage avec la cousine parallèle dans le système arabe », L'Homme, vol. 5, $\mathrm{n}^{\circ}$ 3-4, p. 113-173.

CONTE, E. (1987). «Alliance et parenté élective en Arabie ancienne. Éléments d'une problématique », L'Homme, vol. 27, n 102, p. 119-138.

Der Kaloustian, V., J. Naffah et J. Loiselet (1980). « Genetic diseases in Lebanon », American Journal of Medical Genetics, vol. 7, p. 187-203. 
EL-HAJJ HASSAN, R. (1996). La réalité de l'insertion de la femme dans le marché du travail: une étude du milieu, le village Chmistar. Mémoire de maîtrise, Institut des sciences sociales, Section IV, Ksara, Université libanaise.

El-Khazen, M., G. Kreidy et R. SaAd (1993). Congenital Cardiac Malformations : Statistics and Etiologies. Thèse, Beyrouth, Faculté des sciences médicales, Section II, Université libanaise.

JodeLET, D. (1989). Folies et représentations sociales, Paris, Presses universitaires de France.

KHLAT, M. (1989). Les mariages consanguins à Beyrouth: traditions matrimoniales et santé publique, Paris, Institut national d'études démographiques et Presses universitaires de France.

LAMDOUAR BOUAZZAOUI, N. (1994). " Consanguinité et santé publique au Maroc ", Bulletin de l'Académie nationale de médecine, vol. 178, $\mathrm{n}^{\circ} 6$, p. 1013-1027.

LÉVI-STRAuss, C. (1977). Les structures élémentaires de la parenté, Paris, Presses universitaires de France, coll. «Que sais-je? ».

MANnONI, P. (1998). Les représentations sociales, Paris, Presses universitaires de France.

Mongali, M.A., M. El-Baz, D. Chakroun, H. Jedidi et A. Debbabi (1991). «Étude analytique des cas de diabètes infantiles dans un service de pédiatrie de Tunis », Annales pédiatriques, vol. 38, n 9, p. 623-626.

Moscovicl, S. (1961). La psychanalyse, son image et son public, Paris, Presses universitaires de France.

MustAPHA, M. (1997). Étude éco-génétique des maladies héréditaires de la population du nord du Liban: effets de la consanguinité. Thèse de diplôme d'études approfondies, Tunis, Université de Tunis II.

ORGANISATION MONDIALE DE LA SANTÉ (1993). La prévention de la cécité chez l'enfant, France, OMS. 